\title{
lon beam formation in a very low magnetic field expanding helicon discharge
}

\author{
T. Lafleur, ${ }^{\text {a) }}$ C. Charles, and R. W. Boswell \\ Space Plasma, Power and Propulsion Group, Research School of Physics and Engineering, \\ The Australian National University, Canberra ACT 0200, Australia
}

(Received 15 January 2010; accepted 15 March 2010; published online 13 April 2010)

\begin{abstract}
An ion beam has been measured emerging from a low pressure $(0.04 \mathrm{~Pa})$ helicon plasma reactor over a narrow range of magnetic field values $\left(1 \mathrm{mT}<B_{0}<3 \mathrm{mT}\right)$. The presence of the ion beam occurs simultaneously with a large increase in the plasma density for the same applied magnetic field, produced using a single solenoid half the length of the $m=1 \mathrm{rf}$ antenna. The peak central plasma density of $1.5 \times 10^{17} \mathrm{~m}^{-3}$ is measured to be almost 15 times larger than that occurring before or after the increase, and is associated with a steep axial density gradient which follows the gradient of the magnetic field. During this low magnetic field transition the antenna power transfer efficiency is measured to increase from less than $10 \%$ to $50 \%$, suggesting some form of localized bulk electron heating in the source region associated with the helicon wave. (C) 2010 American Institute of Physics. [doi:10.1063/1.3381093]
\end{abstract}

\section{INTRODUCTION}

The use of diverging magnetic fields in low pressure reactors to create expanding plasmas has recently become an active area of research because of the ion beam that can be created from the associated potential drop. Possible applications range from plasma thrusters in spacecraft to use in the processing industry where a low energy, large area, ion beam is useful for activating surfaces being treated. ${ }^{1}$ The plasma expansion can sometimes arise from geometric effects alone, ${ }^{2}$ but usually requires a diverging magnetic field ${ }^{3-5}$ with typical field strengths of tens of millitesla. ${ }^{6-8}$ Wave heated discharges, ${ }^{9}$ and especially radio frequency (rf) helicon discharges, have attracted much interest over the years, because under certain circumstances it is possible to obtain a high coupling efficiency between the exciting antenna and plasma, and subsequently very high plasma densities can be produced $\left(10^{17}-10^{19} \mathrm{~m}^{-3}\right.$ for powers between a hundred to several kilowatts). ${ }^{10,11}$ In order to achieve these high densities, helicon discharge initiation and wave propagation require an external magnetic field with a typical strength of tens to several hundreds of millitesla. ${ }^{10,11}$ As the magnetic field is increased from zero, a monotonic increase in the plasma density is generally observed, commonly with density jumps that have been associated with mode transitions from capacitive (E) to inductive (H) to wave (W). ${ }^{12}$ These transitions generally appear with the mode being maintained over a broad range of magnetic field values rather than a resonance phenomenon that only occurs at specific magnetic fields. However, at very low fields $\left(B_{0}<5 \mathrm{mT}\right)$, several researchers have reported the existence of a density peak occurring in a helicon discharge over a narrow range of magnetic field values. ${ }^{13-15}$ Sato $^{14}$ obtained densities of 1 $\times 10^{17}-5 \times 10^{17} \mathrm{~m}^{-3}$ for powers between 100 and $2000 \mathrm{~W}$ at $0.05 \mathrm{~Pa}$, while $\mathrm{Chen}^{13}$ reported densities of around 6

${ }^{a)}$ Electronic mail: trevor.lafleur@anu.edu.au. $\times 10^{18} \mathrm{~m}^{-3}$ for $1600 \mathrm{~W}$ at $0.5 \mathrm{~Pa}$ (although this was measured in a small diameter source tube with the density changing by less than some tens of percent). These density peaks are often associated with a corresponding increase in antenna loading resistance, showing that the rf power couples more efficiently into the plasma, ${ }^{15-17}$ and for an excitation frequency of $13.56 \mathrm{MHz}$, usually occur for field strengths of around 2-3 mT. The majority of the work done on low magnetic field helicons has so far focused on the use of uniform fields, and no report has been made of the presence of ion beams in these plasmas. Here we report on a density peak that occurs for a low nonuniform magnetic field produced by a single solenoid $\left(B_{0}<2.5 \mathrm{mT}\right)$, having densities similar to those for uniform fields, ${ }^{14}$ and show that application of the diverging field results in the simultaneous occurrence of an ion beam in the downstream plasma.

\section{EXPERIMENTAL APPARATUS}

The present investigation is carried out in the Piglet helicon reactor shown in Fig. 1. It consists of a double saddle field antenna (approximately extending from $z=-16 \mathrm{~cm}$ to $z=-5 \mathrm{~cm}$ ) surrounding a $20 \mathrm{~cm}$ long, $13.6 \mathrm{~cm}$ internal diameter Pyrex source tube, which is attached to a $28.8 \mathrm{~cm}$ long, $32 \mathrm{~cm}$ diameter aluminum diffusion chamber. The antenna is supplied with $13.56 \mathrm{MHz}$ from a calibrated rf power supply and matching network. Although powers up to $400 \mathrm{~W}$ were investigated, $250 \mathrm{~W}$ is used for all results presented below. One end of the source is terminated by a grounded metal grid to which a turbomolecular/rotary pump system is connected. A pair of solenoids of $\approx 500$ turns each surrounds the source tube/antenna, the solenoid closest to the pump is termed the source coil, while the other is termed the exhaust coil. Argon gas is fed into the reactor via an inlet port in the side of the diffusion chamber, and diagnostic probes (which can translate axially) are inserted into the reactor through ports in the back plate. 


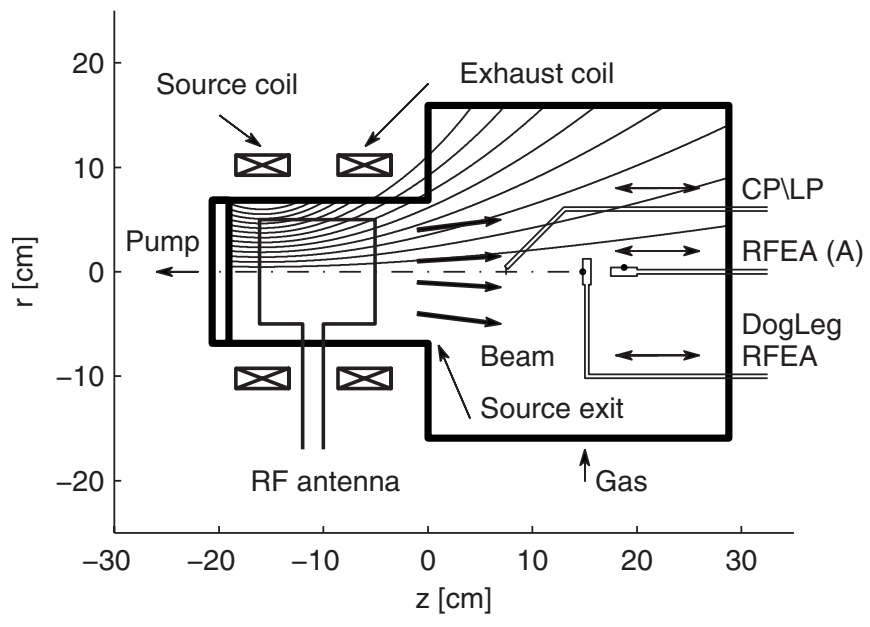

FIG. 1. Schematic of the Piglet reactor showing the plasma source tube, axial solenoids, and diagnostics probes inserted through the backplate. A grounded metal grid separates the pump and source tube. The ion beam location is indicated, together with the magnetic field lines present when the source coil is on (exhaust coil off).

The plasma density is measured with a Langmuir probe (LP) consisting of a $2 \mathrm{~mm}$ diameter nickel disk (attached to the end of a hollow ceramic tube) orientated normal to the axis of the probe. The probe operates in ion saturation mode with an applied bias voltage of $-45 \mathrm{~V}$, and electron temperatures are established using a separate rf compensated Langmuir probe $(\mathrm{CP})$ previously described. ${ }^{18}$ The local plasma potential is obtained from a retarding field energy analyzer [RFEA(A) in Fig. 1] with the orifice facing the walls of the reactor, while a second "DogLeg" RFEA (with a $90^{\circ}$ bend in the support shaft) with orifice facing toward the source region is used to detect the presence of an ion beam. ${ }^{19}$

\section{RESULTS}

A pressure of $0.04 \mathrm{~Pa}$ is investigated because of the large body of experimental data present within the literature for comparison. ${ }^{1,25-8}$ Initial experiments were conducted using both solenoids in order to define the general operating characteristics of the system and to check whether it was behaving in a manner that could be compared with earlier experiments in similar systems. It was found that by keeping the power and pressure constant (at $250 \mathrm{~W}$ and $0.04 \mathrm{~Pa}$, respectively), mode transitions can be induced in Piglet by changing the external applied magnetic field. With the exhaust coil off, an interesting mode transition is observed as the source coil current is varied. Figure 2 shows the density in the center of the source tube $(z=-10 \mathrm{~cm})$ at various radial locations as a function of the maximum magnetic field (with the exhaust coil off). This creates a diverging magnetic field (see Fig. 1) that peaks under the source coil and rapidly decays with axial distance. For low magnetic fields it is found that the density increases by a factor of almost 15 from $1 \times 10^{16}$ to $1.5 \times 10^{17} \mathrm{~m}^{-3}$ over a very narrow range of magnetic field values $\left(1 \mathrm{mT}<B_{0}<3 \mathrm{mT}\right)$, suggesting a direct capacitive (cases A and C on Fig. 2) to wave mode transition ${ }^{20,21}$ (case B on Fig. 2). By contrast, with the exhaust coil on (and with currents greater than about $1 \mathrm{~A}$ ), a transition from a capaci-

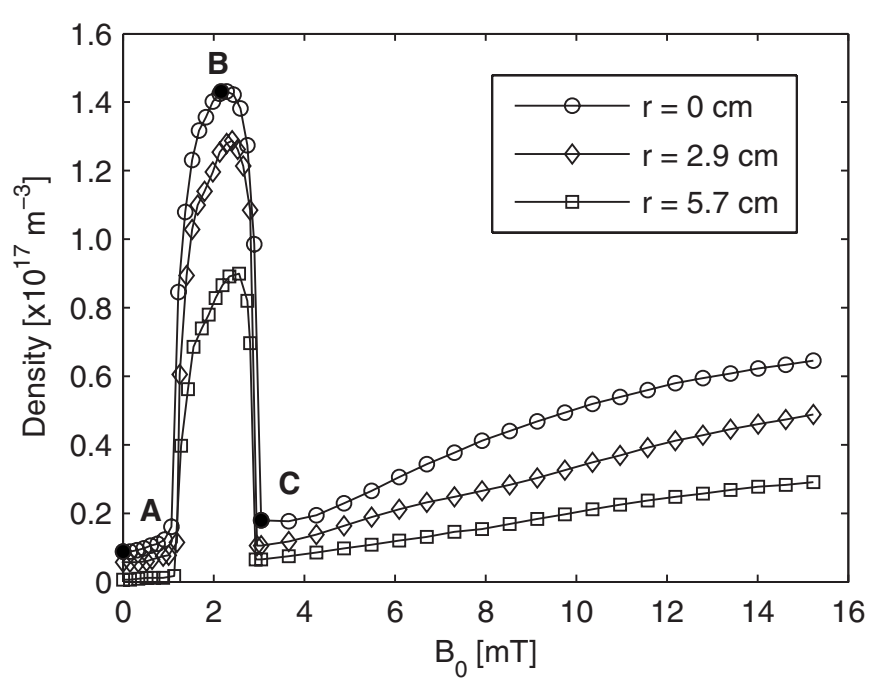

FIG. 2. Plasma density within the source region $(z=-10 \mathrm{~cm})$ at a number of radial locations $(r)$, as a function of the maximum applied magnetic field (as the source coil is varied, with the exhaust coil off). Marked cases A and C correspond to plasma conditions just before and after the observed density peak, while case B corresponds to the maximum of the peak.

tively coupled to an inductively coupled mode is observed as the source coil current is increased (giving magnetic fields greater than around $5 \mathrm{mT}$ ), as has been observed before. ${ }^{5,9,12,22}$ During these transitions the density changes by a much smaller factor $(\approx 2-3)$ and stays high as the magnetic field is further increased.

The axial density profiles for cases $\mathrm{A}-\mathrm{C}$ are shown in Fig. 3 and the radial density profiles at $z=-1 \mathrm{~cm}$ are shown in Fig. 4. These profiles are found using an upstream electron temperature of $9 \mathrm{eV}$ obtained with the compensated probe. For cases $\mathrm{A}$ and $\mathrm{C}$ the axial density remains relatively constant at $1 \times 10^{16}$ and $1.5 \times 10^{16} \mathrm{~m}^{-3}$, respectively, within the reactor. For case B the density is significantly greater

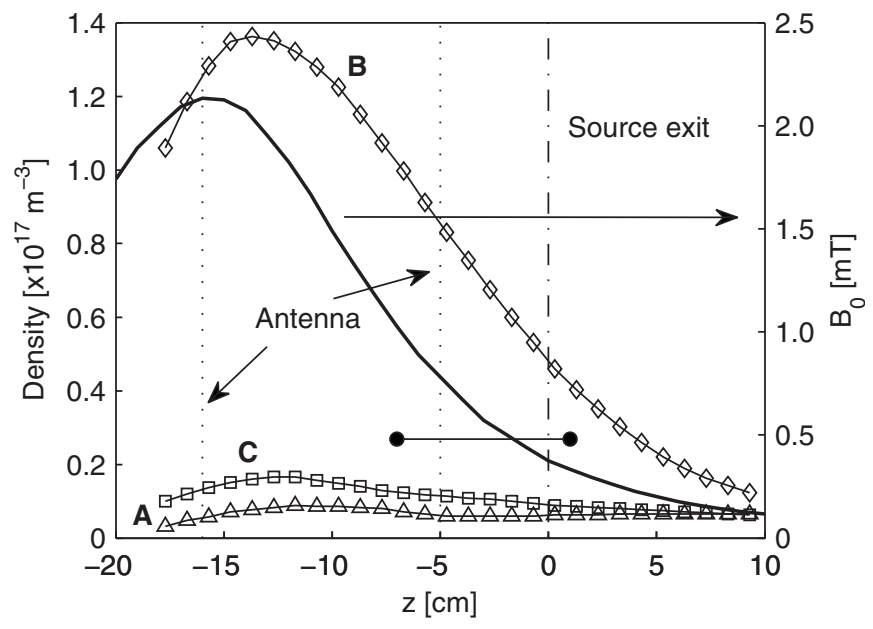

FIG. 3. On-axis plasma density profiles for cases A-C in Fig. 2, with case A (open triangles), case B (open diamonds), and case C (open squares). The dark line shows the measured axial magnetic field for case B. The closed circles and solid joining line shows the location of points with a magnetic field strength of $0.48 \mathrm{mT}$ (giving an electron cyclotron frequency of $13.56 \mathrm{MHz})$ for the cases between the lower (1 mT) and upper ( $3 \mathrm{mT})$ bounds of the density peak in Fig. 2. 


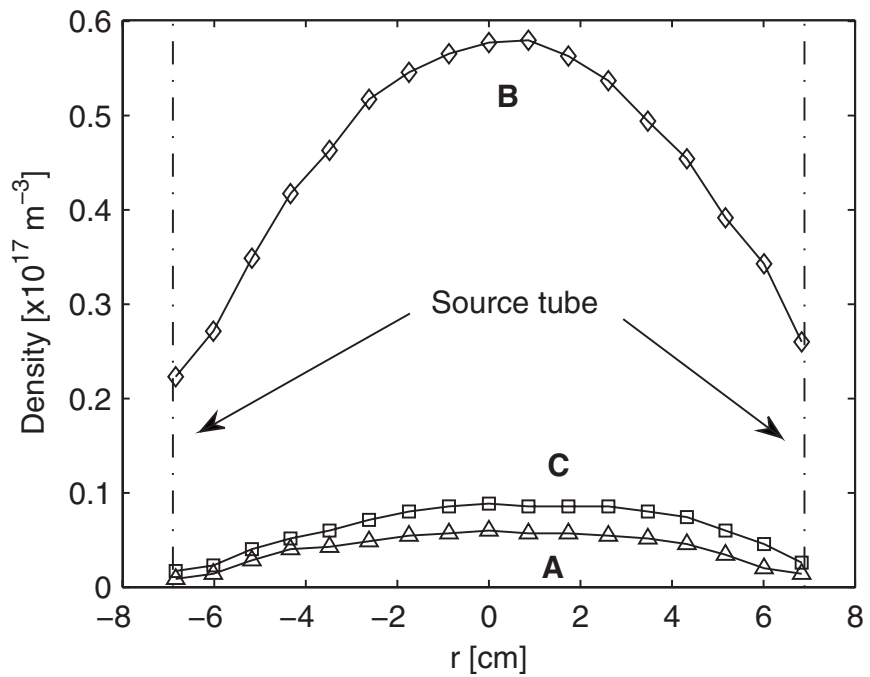

FIG. 4. Radial plasma density profiles at $z=-1 \mathrm{~cm}$ for cases A-C in Fig. 2, with case A (open triangles), case B (open diamonds), and case C (open squares).

throughout the reactor, with a peak value of $1.4 \times 10^{17} \mathrm{~m}^{-3}$ at $z=-13 \mathrm{~cm}$. This is more than an order of magnitude larger than the peak density for cases A and C. Also shown in Fig. 3 is the axial magnetic field variation for case B where it can be seen that the density and magnetic field variations are similar suggesting the plasma is contained in the source tube by the magnetic field. For case A (where no field is present) and case $\mathrm{C}$, the density and magnetic field are not correlated suggesting that the plasma is being created everywhere in the experimental reactor.

Interestingly, during the low magnetic field mode transition shown in Fig. 2, a higher energy ion population (characteristic of an ion beam) is observed in the downstream region. Figure 5 shows the ion energy distribution functions (IEDFs) for cases A and B measured with the DogLeg RFEA at position $z=10 \mathrm{~cm}$ (case $\mathrm{C}$ is similar to case $\mathrm{A}$ and is not shown for clarity). Case A shows only a single peak at the local plasma potential $(\approx 45 \mathrm{~V})$, while case B can be decomposed into two Gaussian ion populations, one centered on the plasma potential $(\approx 26 \mathrm{~V})$ and a second higher energy population at $\approx 40 \mathrm{~V} .{ }^{19}$ It has been previously $\operatorname{shown}^{23}$ that an ion beam is not the only mechanism that can produce a higher energy ion population. If the plasma potential has an rf component (say due to capacitive coupling between the antenna and plasma) then the sheath in front of the grounded RFEA is rf modulated. If the transit time of an ion across this sheath is similar to or less than an rf period, then the measured IEDFs will be affected to some extent by this modulation. In the present situation, the measurement is downstream of the density maximum (see Fig. 3) and the ion transit time is greater than the rf period. An additional experiment can be carried out by changing the orientation of the RFEA which should show no change if there was any rf modulation. RFEA(A) (see Fig. 1) has its orifice facing the walls of the diffusion chamber (i.e., $90^{\circ}$ to the Dogleg RFEA orifice), and when located at $z=10 \mathrm{~cm}$ only a single ion population is present (dark dashed curve in Fig. 5), matching closely to the fitted Gaussian representing the local ion population for the

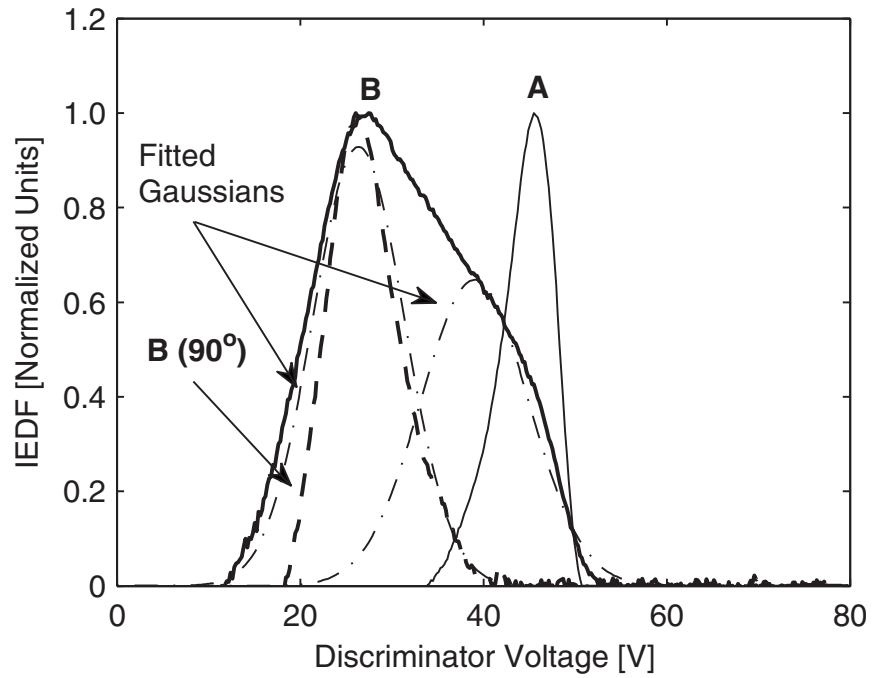

FIG. 5. Normalized IEDFs measured with the "DogLeg" RFEA at position $z=10 \mathrm{~cm}$ for cases A and B in Fig. 2. The light dashed curves show the deconvolution of the IEDF of case B into two Gaussian ion populations, one centered on the local plasma potential (left dashed curve), and a second higher energy ion population (right dashed curve). The dark dashed curve shows the IEDF with the RFEA orifice rotated $90^{\circ}$ to the source tube, obtained with RFEA(A).

Dogleg RFEA at the same location. This is further confirmed by inserting another RFEA into a side port in the diffusion chamber, where the higher energy ion population is only seen with the orifice facing the source region (in particular, disappearing when the RFEA is rotated by $90^{\circ}$ and $180^{\circ}$ ). The above measurements provide strong evidence that the higher energy ion population is not a bimodal distribution due to $\mathrm{rf}$ effects, ${ }^{23}$ but is a directed beam.

Figure 6 shows the axial plasma potential profiles for cases A-C obtained with $\operatorname{RFEA}(\mathrm{A})$. The peak potential of around $40 \mathrm{~V}$ for case $\mathrm{B}$ is lower than the peak potential of about $50 \mathrm{~V}$ for both cases $\mathrm{A}$ and $\mathrm{C}$. Case A shows an axial potential variation that is fairly constant in the diffusion

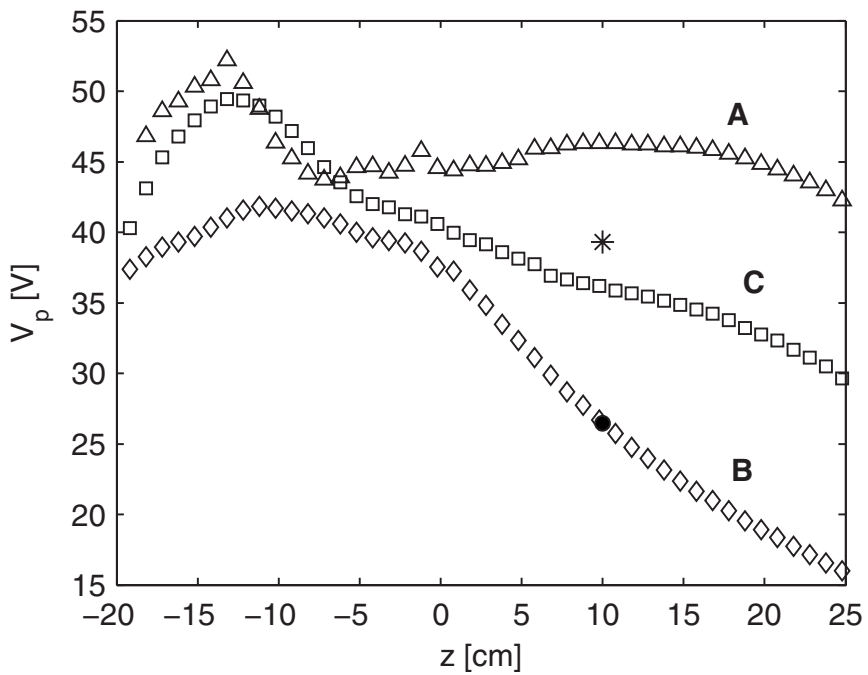

FIG. 6. On-axis plasma potential profiles for cases A-C in Fig. 2, with case A (open triangles), case B (open diamonds), and case C (open squares). The closed circle shows the plasma potential obtained with the "Dogleg" RFEA for case B, while the star shows the ion beam potential (see Fig. 5). 
chamber, with a definite peak at around $z=-12.5 \mathrm{~cm}$ in the source region. A similar peak is present for case C. Case B however shows a potential that remains approximately constant within the source region, before rapidly decaying for $z>0 \mathrm{~cm}$. From Fig. 5 the ion beam energy for case B is approximately $39 \mathrm{~V}$, which is $13 \mathrm{~V}$ above the local plasma potential of $26 \mathrm{~V}$, and well correlated with the peak upstream potential of $41 \mathrm{~V}$ in Fig. 6.

Based on the above results, the system seems to transition from an initially axially uniform plasma state to an expanding plasma (containing an ion beam) and then back to a more or less uniform plasma state. In a previous publication ${ }^{5}$ a transition was reported at $\approx 5 \mathrm{mT}$ together with the observation of a higher energy ion population. It was suggested that as the magnetic field was increased, the ion gyroradius decreased until at around $5 \mathrm{mT}$, the gyroradius was equal to the source tube diameter. Further increases in the magnetic field would decrease the gyroradius even more resulting in a decrease in the radial plasma loss rate and a subsequent increase in the plasma density, as was observed. In the present case however, a transition occurs at a much lower magnetic field $(1 \mathrm{mT})$ with the peak at $\approx 2 \mathrm{mT}$, after which further increases in the magnetic field result in a decrease in the plasma density. Using a similar ion temperature to Ref. 5 of $0.2 \mathrm{eV}$, we obtain an ion gyroradius of $14.6 \mathrm{~cm}$ at $2 \mathrm{mT}$, well above the source tube radius of $6.8 \mathrm{~cm}$. Since plasma confinement would be expected to increase as the magnetic field increases, the present behavior cannot be the result of the same physics as the transition previously observed.

Since the antenna/plasma is matched at each magnetic field setting in Fig. 2, it is important to investigate how the plasma density can increase by such a large factor, given that the plasma potential does not change significantly, and that improved plasma confinement apparently cannot explain the observed density peak. To do this we calculate the effective resistance of the antenna/plasma system. A calibrated rf current probe is placed around one of the antenna feedthroughs and is used to measure the current in the antenna, while the forward and reverse power are determined from the calibrated $\mathrm{rf}$ power supply. The effective antenna resistance, $R$, can then be found from $P=I_{\mathrm{rms}}^{2} R$, where $I_{\mathrm{rms}}$ is the root mean square antenna current, and $P$ is the net input power to the antenna/matchbox circuit (forward minus reverse power). The effective antenna resistance during the transition is shown in Fig. 7 where it can be seen that a peak in the resistance occurs, well correlated with the density peak in Fig. 2. The power transfer efficiency, $\eta$, can be obtained from $\eta=R_{p} / R$, where $R_{p}=R-R_{\text {ext }}$ is the effective plasma resistance, ${ }^{17}$ and $R_{\text {ext }}$ is the antenna/matchbox circuit resistance $(0.25 \Omega$ for Piglet). This gives efficiencies of $5 \%$, $50 \%$, and $10 \%$ for cases $\mathrm{A}, \mathrm{B}$, and $\mathrm{C}$, respectively, showing that more power is deposited into the plasma for case $\mathrm{B}$, thus offering an explanation as to why the density can increase by such a large factor during the transition in Fig. 2. The details of how the power is transferred to the electrons (and hence the plasma) are, however, not yet understood.

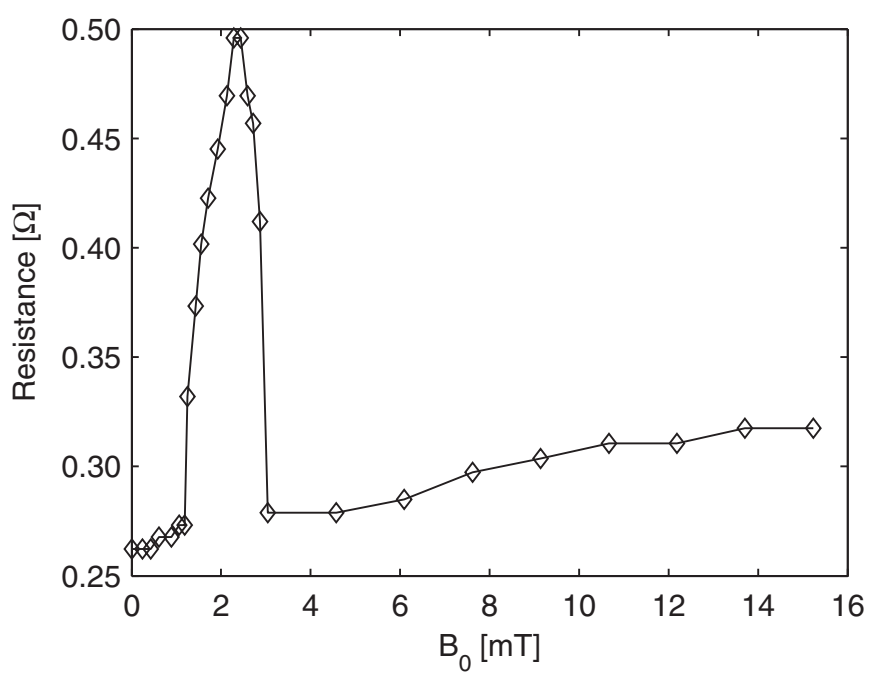

FIG. 7. Measured effective antenna resistance as a function of the maximum applied magnetic field for the transition shown in Fig. 2.

\section{DISCUSSION}

There are a number of publications that show that the axial plasma density in short helicon (or inductively coupled) systems tend to follow the axial magnetic field variation when this is sufficiently large ( $>10 \mathrm{mT}$ or so). ${ }^{5,19}$ This has been measured using Langmuir probes and optical emission spectroscopy. At lower plasma densities where the skin depth is greater than a typical system dimension, large capacitive rf electric fields will accelerate electrons throughout the volume irrespective of the shape of the magnetic field. For the present experiment, case B resembles a region of inductively or wave coupled plasma that exists in the cavity formed by the magnetic field in the source tube, whereas cases A and C resemble capacitively coupled plasmas. It is not yet clear how this can occur, nor what causes the observed density peak.

On the one hand the mode transition shows similar characteristics to the low field density peaks reported before, where it has been suggested that wave reflection at the boundaries causes constructive interference enhancing the wave fields, ${ }^{16,17}$ but how this improves the electron heating process remains unclear. The source region in Piglet is terminated by a grounded metal grid, so that wave reflections could indeed occur here (although $\mathrm{Cho}^{17}$ has shown that for an $m=1$ antenna, such as is used here, a density peak can be formed regardless of reflections). Additionally the peak density and range of magnetic field values over which the density peak occurs at in the present case are consistent with the literature. On the other hand, the axial magnetic field for cases between the lower $(1 \mathrm{mT})$ and upper $(3 \mathrm{mT})$ bounds of the density peak in Fig. 2 (and hence case B) decays to a value of $0.48 \mathrm{mT}$ close to the source tube exit (see Fig. 3). This yields an electron cyclotron frequency of $13.56 \mathrm{MHz}$, which is equal to the applied antenna frequency. Thus an electron cyclotron resonance region exists within the system. This suggests that ECR damping of the right hand polarized helicon wave could be playing a role in the mode transition; an effect that would be absent from the low magnetic field 
density peaks reported before for uniform fields. Observation of the axial density profile for case B in Fig. 3 shows that no significant features are present near this ECR region, however, due to the low operating pressure used it is likely that nonlocal heating of the electrons is occurring so that the density peak appears far from the heating region. More detailed studies of the above mechanisms are currently being undertaken.

\section{SUMMARY AND CONCLUSIONS}

A large density peak has been observed in the presence of a very low magnetic field together with the simultaneous measurement of an ion beam in the downstream plasma. The density peak is associated with both an increased power coupling efficiency between the antenna and plasma, and a transition to an expanding plasma wherein which the ion beam is formed.

${ }^{1}$ C. Charles, J. Phys. D 42, 163001 (2009).

${ }^{2}$ C. S. Corr, J. Zanger, R. W. Boswell, and C. Charles, Appl. Phys. Lett. 91, 241501 (2007).

${ }^{3}$ F. F. Chen, Phys. Plasmas 13, 034502 (2006).

${ }^{4}$ A. Fruchtman, Phys. Rev. Lett. 96, 065002 (2006).
${ }^{5}$ C. Charles and R. W. Boswell, Appl. Phys. Lett. 91, 201505 (2007).

${ }^{6}$ C. Charles and R. W. Boswell, Appl. Phys. Lett. 82, 1356 (2003).

${ }^{7}$ M. D. West, C. Charles, and R. W. Boswell, J. Propul. Power 24, 134 (2008).

${ }^{8}$ K. Takahashi, K. Oguni, H. Yamada, and T. Fujiwara, Phys. Plasmas 15, 084501 (2008)

${ }^{9}$ M. A. Lieberman and A. J. Lichtenberg, Principles of Plasma Discharges and Materials Processing (Wiley, New Jersey, 2005).

${ }^{10}$ R. W. Boswell and F. F. Chen, IEEE Trans. Plasma Sci. 25, 1229 (1997).

${ }^{11}$ F. F. Chen and R. W. Boswell, IEEE Trans. Plasma Sci. 25, 1245 (1997).

${ }^{12}$ A. R. Ellingboe and R. W. Boswell, Phys. Plasmas 3, 2797 (1996).

${ }^{13}$ F. F. Chen, J. Vac. Sci. Technol. A 10, 1389 (1992).

${ }^{14}$ G. Sato, W. Oohara, and R. Hatakeyama, Plasma Sources Sci. Technol. 9, 3171 (2002).

${ }^{15}$ S. J. Wang, J. G. Kwak, C. B. Kim, and S. K. Kim, Phys. Lett. A 313, 278 (2003).

${ }^{16}$ F. F. Chen, Phys. Plasmas 10, 2586 (2003)

${ }^{17}$ S. Cho, Phys. Plasmas 13, 033504 (2006).

${ }^{18}$ K. Takahashi, C. Charles, and R. W. Boswell, Phys. Plasmas 14, 114503 (2007).

${ }^{19}$ T. Lafleur, C. Charles, and R. W. Boswell, Phys. Plasmas 16, 044510 (2009).

${ }^{20}$ A. Perry, G. Conway, R. W. Boswell, and H. Persing, Phys. Plasmas 9, 3171 (2002).

${ }^{21}$ C. M. Franck, O. Grulke, and T. Klinger, Phys. Plasmas 10, 323 (2003).

${ }^{22}$ M. A. Lieberman and R. W. Boswell, J. Phys. IV 8, 145 (1998).

${ }^{23}$ C. Charles, A. W. Degeling, T. E. Sheridan, J. H. Harris, M. A. Lieberman, and R. W. Boswell, Phys. Plasmas 7, 5232 (2000). 